\title{
What we've learned so far about ADT
}

\author{
Neil E. Fleshner, MPH, MD, FRCSC
}

Division of Urology, University Health Network, University of Toronto, Toronto, ON

Cite as: Can Urol Assoc J 2014;8(7-8):S133. http://dx.doi.org/10.5489/cuaj.2283 Published online August 11, 2014.

A Ithough the use of androgen deprivation has been with us for over 60 years, we continue to learn more and more about the androgen axis, how best to suppress it, and how to maximize the metabolic challenges that it poses for our patients. This year's American Urological Association (AUA) meeting again provided valuable insights into some key aspects of androgen axis targeting. The emerging data of cardiovascular risk, not only at baseline but also as a consequence of therapy, continue to gain momentum; urologists need to understand these data in a broader context of preventative strategies.

The emerging role for degarelix as a safer agent for these patients is gaining increased notice among the urological community. The novel observations and scientific data on follicle-stimulating hormone (FSH) and its putative important functions are beginning to gain traction in the scientific space. The McMaster data presented at the 2014 meeting adds to this body of knowledge as it appears that at least part of the deleterious metabolic effects of androgen deprivation therapy (ADT) may lie in its FSH-suppressing activity and not all due to lowering of testosterone. This observation, in mice only, may open novel opportunities for therapeutic intervention. Furthermore, the National Cancer Institute of Canada (NCIC) PR-7 data confirm, with the best available cohort to date, that nadir testosterone levels do affect outcomes in men on ADT. Up to this date, most felt that the cohort-type studies conducted in Europe were small and frankly hypothesis-generating. The data by Klotz and colleagues, however, challenge these views and reinforce that testosterone levels during ADT should be measured in conjunction with prostate-specific antigen. The therapeutic options and "what can we do about it" factors need to be sorted out in well-conducted clinical trials.

I believe we may be on the verge of reintroducing ADT in high-risk populations, not only with radiation patients but also with surgical ones. We may revisit the paradigm that seemed to be closed 15 years ago because of the results of a host of novel neoadjuvant trials. In my view, past mistakes in trial design, a re-examining of older data, and better androgen-axis-targeting agents may make these questions relevant again. Suffice it to say, "we never stop learning!"

Competing interests: Dr. Fleshner is a member of the Advisory Board for Amgen, Janssen, Astellas and Eli Lilly. He has received honoraria from Amgen, Janssen, Astellas and Eli Lilly. He is and has participated in clinical trials for Amgen, Janssen, Medivation, OICR, and Prostate Cancer Canada.

This paper has been peer-reviewed.

Correspondence: Dr. Neil Fleshner, 610 University Ave., Suite 3-130, Toronto ON M5G 2M9. neil.fleshner@uhn.on.ca 